\title{
Birth order as a source of diversification in spring phenology and its potential effects on performance in greater duckweed, Spirodela polyrhiza
}

By

Riley S. Morris

\begin{abstract}
A thesis submitted to the Faculty of Graduate
and Postdoctoral Affairs in partial fulfillment of the

requirements for the degree of
\end{abstract}

\author{
Master of Science \\ In \\ Biology \\ Carleton University \\ Ottawa, Ontario
}

(C) 2018

Riley S. Morris 


\begin{abstract}
Organisms must be able to adapt in order to persist in dynamic and unstable environments. Now, in the face of rapid environmental change, questions about plants' intrinsic tolerance to variability and unpredictable environments are especially relevant. The timing of both winter freeze-up and spring thaws are unpredictable and under these variable environments, clonal populations of Spirodela polyrhiza (greater duckweed) provide an excellent case study of phenotypic diversification as a risk aversion strategy. Previous research on this species has demonstrated that potential diversification bet hedging in the phenology of the production of turions-the quiescent overwintering structure that rests at the sediment surface-is generated by birth order within clones. Like production of turions which occurs in the fall, the timing of turion reactivation the following spring may also have profound fitness consequences due to the risk of the thaw and re-freezing of the water's surface. I therefore hypothesize that variance in turion reactivation phenology within clones is influenced by birth order of turions. This was tested through a laboratory study that determined the source of observed phenological variability, and an outdoor mesocosm study that further examined fitness consequences of variance in the timing of turion reactivation under different temperature treatments. The results showed that both temperature and birth order play a role in reactivation timing in that early birth order turions generally reactivate before later birth orders. The effect size of this birth order interaction was found to decrease with temperature. Furthermore, although an effect of turion birth order on lineage performance was not seen, temperature was shown to be positively associated with performance. This research suggests mechanisms whereby clones may generate diversification strategies. If various phenotypes (birth orders) perform differently depending on the environment, a "phenotype-by-environment interaction" generates potential diversification bet hedging. Such diversification could mitigate impacts of both seasonal unpredictability and larger scale climate trends.
\end{abstract}




\section{Acknowledgements}

This thesis could not have been done without the help and support of a number of individuals. I would first like to thank my thesis supervisor Dr. Andrew Simons, who has been a wonderful research supervisor and mentor and has endlessly supported me throughout my studies in his lab. His expertise in research and writing have been a great example for me as I navigated my way through my degree program. Along with Dr. Simons, I would like to send my deepest thanks to my thesis advisory committee Dr. Jessica Forrest and Dr. Tom Sherratt, who have been excellent in helping provide additional guidance. I would also like to thank Dr. Naomi Cappuccino and Dr. Joseph Bennett for graciously agreeing to act as internal examiner and chair to my defence. I would like to acknowledge the financial support received from Dr. Simons' NSERC Discovery Grant and from the Faculty of Graduate Studies.

I would like to thank Vivienne Seymour and Mahmoud El-Saadi for their generous contributions to my research. My daughter was born mid-way through the last year of my degree and Dr. Simons' understanding combined with Vivienne and Mahmoud's research assistance made completing my thesis possible. I am forever indebted to you and could not thank you enough. I would also like to thank Hebah Mejbel for her knowledgeable consultation on duckweed and experimental design as well as her help in growing my test population. Additionally, Ryan Chlebak contributed a lot to the initial development of the mesocosm system by building the control shed, helping to prepare the garden for the mesocosm array, helping with the initial setup of the data logger system, and helping keep the growth chambers online. The Simons Lab banded together to help construct over 500 rafts for use in the mesocosm study, this was a serious undertaking and I would like to express my deepest thanks to Lina Wen, Shravan Raghu, Harry Hitsman, Winston Campeau, Ryan Chlebak, and Mahmoud El-Saadi for their help in raft construction. I would also like to thank the Simons Lab as well as Felipe Dargent, Karl Loeffler-Henry, and James McLaren for their fruitful discussion and advise during our weekly lab meetings. Over the past 
two years I have been given a lot of flexibility from a Dr. Simons, who has been generous in supporting me after the birth of my daughter and during my extension semester. Furthermore, my employer Jp2g Consultants Inc., has been very generous in allowing me to prioritize my schooling while maintaining my place within their company.

I would like to express my thanks to my family, Judi, Gerry, Jessica, Brandon, and Robyn for their love and support throughout my program. Moreover, my daughter, though to young to know it, has inspired me every day to do my best and to achieve something that she will one day be proud of. I would finally like to thank my loving wife, Cassandra Morris, who has given me her fullest support throughout the entirety of my degree. She has been by my side through the highs and lows and has always helped to motivate me when I need it most. More than this, she has also helped me with data input and counting and photographing duckweed. She has also helped me rationalize arguments during the writing process and helped with thesis editing. Your love and support have been paramount. 
Table of Contents

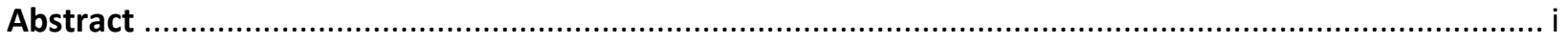

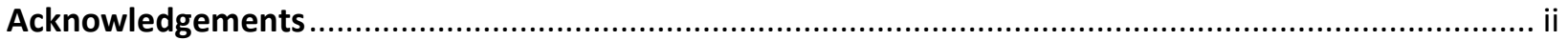

Chapter 1: General Introduction

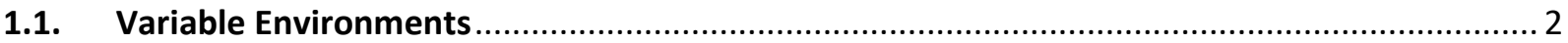

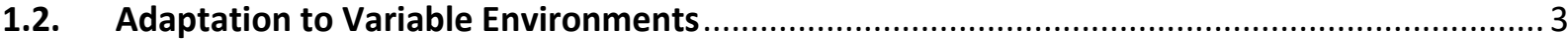

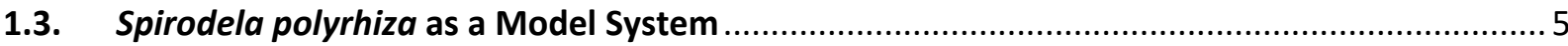

Chapter 2: Birth Order as a Source of Phenological Variation and its Associated Fitness Implications in the Duckweed, Spirodela polyrhiza

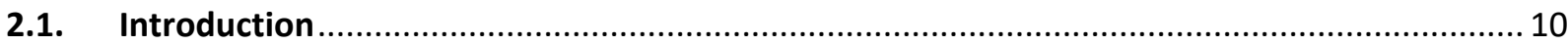

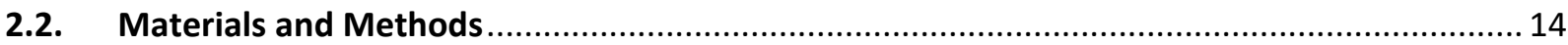

2.2.1. Growth and Maintenance of the Population .................................................... 14

2.2.2. Laboratory Study of Turion Reactivation Time .............................................. 15

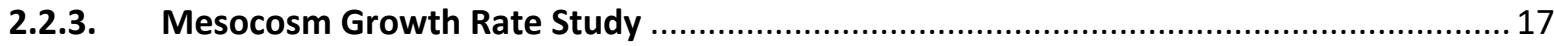

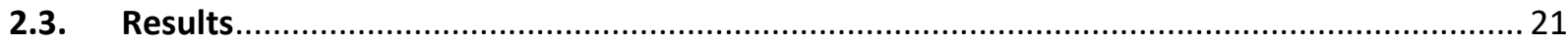

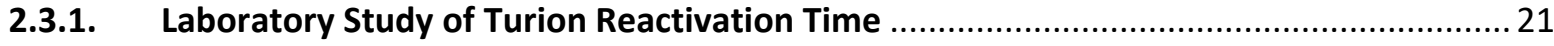

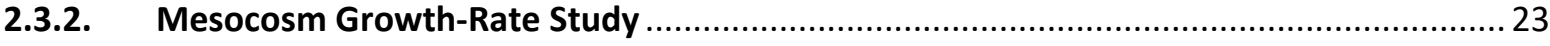

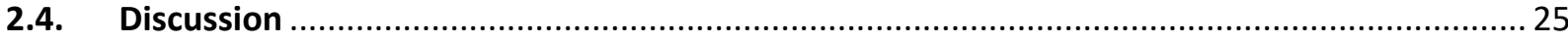

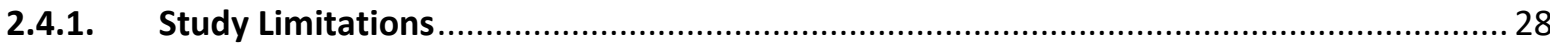

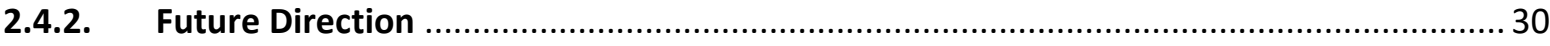

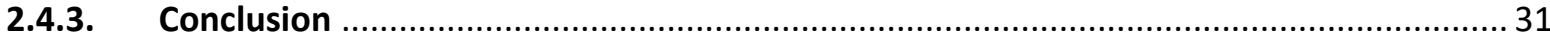

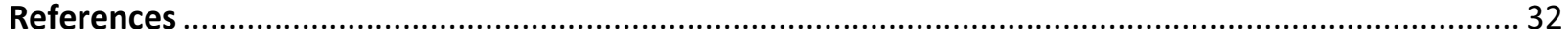




\section{List of Appendices}

Appendix A Photographs of Equipment and Apparatus

Appendix B

R Script for the Laboratory Reactivation Study

Appendix C

R Script for the Mesocosm Study

Appendix D

Mesocosm Control Program for Campbell Scientific CR6 Data Logger

Appendix E

Additional Genotype Analysis

Appendix F

Environmental Data from the Mesocosm Study

\section{List of Tables}

Table 2.1: Fully-factorial ANOVA for effects of reactivation temperature, mother birth order, and

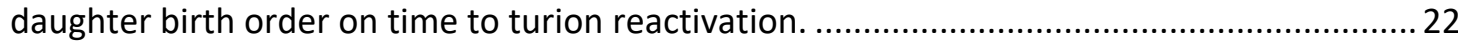

Table 2.2: ANOVA for frond growth rate measured as change in dry biomass ......................................24

\section{List of Figures}

Figure 2.1: Turion yield for reactivation study for combinations of turion birth orders and mother birth orders

Figure 2.2: Turion yield for growth rate study for combinations of turion birth orders and mother birth orders

Figure 2.3: Overall reactivation timing of S. polyrhiza turions in the lab for the three temperature treatments

Figure 2.4: Reactivation time of S. polyrhiza turions across turion and mother birth orders for the three temperature treatments

Figure 2.5: Growth rate of S. polyrhiza as a function of temperature treatment .................................. 25

Figure 2.6: Predicted growth rate response to turion birth order and treatment effect. 28 
CHAPTER 1

GENERAL INTRODUCTION 


\subsection{Variable Environments}

Environments are dynamic and can vary both spatially (Seger and Brockmann 1987; Morrongiello et al. 2012; Chen et al. 2018) and, more relevant to this study, temporally (Cohen 1966; Seger and Brockmann 1987; Simons and Johnston 2003). Changes in temporally variable environments include (but are not limited to) biotic fluctuations, as well as abiotic fluctuations in temperature, precipitation, and photoperiod (Childs et al. 2010; Brewer et al. 2017). These abiotic environmental changes are comprised of deterministic and stochastic components (Graham et al. 2014; Botero et al. 2015; Mejbel and Simons 2018) and can lead to climate differences across the globe; for example, semi-regular wet-dry cycles near the equator, cold winters and warm summers in the mid-latitudes, and mild bright summers and cold dark winters near the poles.

Deterministic changes in the environment are generally predictable and include rhythmic oscillations, such as the non-random component of seasonal change (Wang and Dillon 2014; Botero et al. 2015). While seasonal change is generally deterministic, interannual variation in the timing and mangitude of environmental change can be highly stochastic (Proulx and Day 2002; Wang and Dillon 2014; Botero et al. 2015; Noh et al. 2017); for example, the timing of first snow melt in higher latitudes can vary greatly from year to year (Van Vuren and Armitage 1991; Stinson 2004). This is the unpredictable component of environmental variation and it presents a challenge for organismal survival (Simons 2014; Furness et al. 2015; Rossi et al. 2017; Tarazona et al. 2017).

Fitness, and thus adaptation, is dependent on interactions between phenotype and environmental changes. However, because phenologies are expected to be "tuned" to specific environments and to the phenology of other members of their community (both inter- and intraspacific coordination) (Lieth 1974; Edwards et al. 2004; Franks et al. 2007; Richardson et al. 2013, 2016; Carter et al. 2018; Wadgymar et al. 2018), an organism's interaction with its environment can be quite complex. A rapidly changing environment can cause organisms to shift their phenological schedules and thus create 
phenological suboptimality and mistimings at the community level (Edwards et al. 2004; Franks et al. 2007; Carter et al. 2018; Wadgymar et al. 2018).

Our changing climate is speculated to exacerbate this issue by increasing the rate and propensity of both predictable and unpredictable components of change (Stocker et al. 2013; Wang and Dillon 2014; Botero et al. 2015; Noh et al. 2017). Organisms experiencing rapid change beyond their plastic tolorance must either adapt (Umina et al. 2005; Norberg et al. 2012) or shift their geographical range (Macel et al. 2017; Archambault et al. 2018), else they face potential extirpation or extinction (Norberg et al. 2012; Wiens 2016; Saatkamp et al. 2018).

\subsection{Adaptation to Variable Environments}

Under temporally variable conditions, fluctuations in the magnitude and/or direction of selection cause the local trait optima to shift, sometimes unpredictably (Bell and Collins 2008; Simons 2009; Bonnet and Postma 2018). However, when the timescale of environmental fluctuation is large relative to an organism's generation time, the rate of response to natural selection on mutational and standing genetic variation in the population may be sufficient to allow adaptation to the environmental change (Bell and Collins 2008; Simons 2011; Botero et al. 2015). This is referred to as adaptive tracking and it is dependent on the availability of standing genetic variation within the population (Barrett and Schluter 2008; Bell and Collins 2008; Simons 2011).

A lag in response to selection through adaptive tracking is inevitable; immediate response, even in populations with nonoverlapping generations, would require that all phenotypic variance in a population be generated by additive genetic variance (Turelli 1984; Schiffers et al. 2013). If the rate of environmental change outpaces the "tracking" abilities of an organism, the population would suffer low absolute fitness. At the extreme limits of tracking, evolutionary rescue is said to occur when mean population fitness has declined, and novel genetic variation allows population recovery (Bell and Collins 2008; Simons 2011; Christie and Searle 2018); without this variation the fate would be local extinction. 
The efficacy of adaptive tracking is thus limited not only by the availability of genetic variation, but by the severity and rate of environmental change. The literature notes two other modes of adaptation to variable environments: phenotypic plasticity (Via and Lande 1985; Bell and Collins 2008; Simons 2011; Botero et al. 2015; Mejbel and Simons 2018) and bet hedging (Seger and Brockmann 1987; Philippi and Seger 1989; Bell and Collins 2008; Simons 2011; Starrfelt and Kokko 2012; Botero et al. 2015; Mejbel and Simons 2018). It should be noted that these modes of response also evolve through adaptive tracking but over longer time scales (Simons 2011) even if they appear to "prepare" for future environments. Adaptive phenotypic plasticity is expected to evolve when environmental cues are reliable predictors of the future state of the environment (Schlichting and Pigliucci 1998; van Kleunen and Fischer 2005; Simons 2011; Furness et al. 2015; Maxwell and Magwene 2017). Adaptive plasticity is the differential expression of a phenotype across environments and provides a fitness benefit to the individual (Bradshaw 1965; Via and Lande 1985; Scheiner 1993; Simons 2011). In contrast to adaptive plasticity, bet hedging traits are expected to evolve when environmental cues are not predictive of future conditions (Simons 2011; Furness et al. 2015; Maxwell and Magwene 2017), or when tracking and plasticity are constrained (Simons 2011). Bet hedging strategies trade-off arithmetic mean, or "expected fitness" for reduced fitness variance, thus increasing geometric-mean fitness (Cohen 1966; Seger and Brockmann 1987; Philippi and Seger 1989; Simons 2009, 2014; Childs et al. 2010; Starrfelt and Kokko 2012; Graham et al. 2014; Crowley et al. 2016). Bet hedging may take two forms: conservative and diversification. Some researchers identify these two forms as distinct "types" of bet hedging (as in Seger and Brockmann 1987), where others (Starrfelt and Kokko 2012) view them as two extremes of a continuum. Conservative bet hedging is defined as the expression of a suboptimal but "safe" trait value that reduces fitness variability over the long-term (Seger and Brockmann 1987; Philippi and Seger 1989; Childs et al. 2010; Simons 2011; Botero et al. 2015; Maxwell and Magwene 2017). The alternative, diversification bet hedging, is when a genotype reduces variability in fitness by spreading risk of failure 
among multiple phenotypes, expressed simultaneously (Seger and Brockmann 1987; Philippi and Seger 1989; Childs et al. 2010; Simons 2011; Botero et al. 2015; Maxwell and Magwene 2017).

As an example, studies on the obligately self fertilizing monocarpic perennial, Lobelia inflata, have found that the high degree of phenotypic variability in both the germination fraction and the timing of germination (Simons and Johnston 2006) increase fitness under the extent of environmental variance observed (Simons and Johnston 2006; Simons 2009, 2014). Furthermore, suboptimality in the bolting and subsequent flowering schedule of $L$. inflata suggests the expression of conservative bet hedging that maximizes fitness under rare, short season length (Simons and Johnston 2003). In both cases, results show that the bet hedging traits in this species operate along a norm of reaction, implying the joint expression of adaptive phenotypic plasticity and bet hedging (Simons 2014). These studies argue that the expression of these adaptive traits allows $L$. inflata to persist through variable and unpredictable environments with little to no genetic variation among offspring. Similarly, putative diversification bet hedging has been observed in clone populations of the floating aquatic plant Spirodela polyrhiza (L.) Schleiden, which despite genetic homogeneity, produces offspring exhibiting diverse phenotypes (Compton 2013; Mejbel and Simons 2018). This quality of Spirodela polyrhiza will be the focus of this research.

\subsection{Spirodela polyrhiza as a Model System}

Turions (quiescent overwintering bodies) of the duckweed species, S. polyrhiza, are paramount to their survival under fluctuating conditions (Jacobs 1947; Appenroth et al. 1996; Oláh et al. 2017) and make them an ideal model system for studies on adaptation in variable environments. Furthermore, duckweed species are among the fastest growing angiosperms in the world in terms of biomass production (Kutschera and Niklas 2015). They have been the focus of studies in physiology (Jacobs 1947; Perry 1968; Appenroth et al. 1989, 1996; Ley et al. 1997; Lemon et al. 2001), toxicology (Appenroth et al. 2000; Oláh et al. 2015, 2018; Geng et al. 2018; Singh et al. 2018), and evolutionary biology (Vasseur and 
Aarssen 1992; Barks and Laird 2015, 2016; Ankutowicz and Laird 2018; Mejbel and Simons 2018) as well as in industrial contexts with applications in wastewater treatment (Wang et al. 2014; Ziegler et al. 2017; Kaur et al. 2018; Singh et al. 2018), pharmaceuticals (Wang et al. 2014), human consumption (Wang et al. 2014; Appenroth et al. 2017; Kaur et al. 2018), and biofuel crop production (Wang et al. 2014; Gönen 2018; Kaur et al. 2018). Duckweed, although sometimes still considered to form the family Lemnaceae, are now more commonly accepted as belonging to the subfamily Lemnoideae within the family Araceae (Appenroth et al. 1996; Appenroth 2003; Wang et al. 2014; Oláh et al. 2017). Spirodela polyrhiza is the most studied turion forming aquatic plant (Oláh et al. 2017) and is one of 35 described species of duckweed, 15 of which are known to produce turions as vegetative overwintering organs (Jacobs 1947; Perry 1968; Appenroth et al. 1996; Appenroth 2003; Oláh et al. 2015, 2017; Mejbel and Simons 2018), which are a central focus of this study.

Although S. polyrhiza is a monoecious (cosexual) angiosperm and is facultatively sexual, sexual reproduction is rarely documented and typical reproduction occurs through the asexual budding of fronds, or when prompted, turions (Jacobs 1947; Kutschera and Niklas 2015). Asexual budding occurs from meristematic pockets located at the proximal end of the frond (Jacobs 1947; Appenroth 2002a; Oláh et al. 2014; Mejbel and Simons 2018). Daughter fronds are tethered to their mothers via stipules that eventually break, forming new duckweed rafts (Wang et al. 2014).

Turions form from the same meristematic pockets as normal fronds (Jacobs 1947; Wang et al. 2014). Environmental cues that are known to trigger the switch to formation of turions include reduced temperature (Jacobs 1947; Perry 1968; Appenroth et al. 1996; Ley et al. 1997; Appenroth 2002a,b; Oláh et al. 2017; Mejbel and Simons 2018), nutrient limitation (Jacobs 1947; Appenroth et al. 1989; Appenroth 2002b), and contaminant exposure (Appenroth et al. 2000; Oláh et al. 2014, 2015). This paper will focus on populations found in temperate climates that exhibit turion formation behaviour as a means of survival through winter months that would otherwise be lethal (Adamec 2004). 
Turions are dense structures that lack aerenchyma and are rich in starch; they sink to the sediment surface following formation (Jacobs 1947; Mejbel and Simons 2018). Turions are initially in a state of innate dormancy, which breaks following a short cold (or "after-ripening") period at which time they enter an imposed dormancy (Jacobs 1947; Ley et al. 1997; Adamec 2004; Oláh et al. 2017). Under this latter state of dormancy, turions are no longer endogenously inhibited from reactivating (parallel to germination in seeds), but resist until external inhibitors (i.e. adverse environmental conditions) are lifted (Appenroth et al. 1996; Ley et al. 1997; Adamec 2004; Oláh et al. 2017). It is proposed that the evolutionary driver of this dormancy structure is to prevent turions from reactivating before winter (and therefore dying) if laid down earlier in the growing season (Appenroth et al. 1996).

When dormancy requirements of turions have been met and the environment is conducive, metabolic processes increase, fuelled by the high starch reserves and other carbohydrates (Ley et al. 1997). $\mathrm{A} \mathrm{CO}_{2}$ bubble is produced by the turion through cellular respiration and trapped, propelling the turion to the water's surface where it buds a new daughter frond (Oláh et al. 2017). The control of turion reactivation currently remains under-studied; however, research indicates that it is related to red light and temperature cues following after-ripening (Jacobs 1947; Appenroth et al. 1996; Oláh et al. 2017). In this study, an exploratory laboratory test showed that turions do not readily reactivate below $5^{\circ} \mathrm{C}$ while exposed to light.

In addition to cue response, frond and turion behaviour can be influenced by phenotypic differences among individuals. Recent studies on senescence in duckweed have found that as a mother frond ages, its offspring decline in both size and fitness (Barks and Laird 2015, 2016; Ankutowicz and Laird 2018). This progressive decline in offspring "quality" produces differences among fronds of different birth order (Barks and Laird 2016). Moreover, Mejbel and Simons (2018) found that differences in birth order generate variability in the timing of turion formation, potentially acting as a diversification bet-hedging strategy. This thesis will focus on unanswered questions regarding the effects of birth order 
on variability in the timing of spring reactivation and its resulting fitness implications. I begin by asking, whether mother and turion birth order variation of overwintering turions is a source of phenological variation within clones in spring emergence. I then ask whether fitness consequences of potential diversification in timing among birth orders is further complicated by variation in temperature-specific performance among birth orders over a range of temperatures. This potential phenotype-byenvironment interaction could be a source of diversification bet hedging that reduces a genotype's fitness variation among generations within clones. Mechanisms by which clone populations adapt to variable environments are understudied; this thesis helps to expand our knowledge in this area. 
CHAPTER 2

BIRTH ORDER AS A SOURCE OF PHENOLOGICAL VARIATION, AND ITS ASSOCIATED FITNESS IMPLICATIONS IN THE DUCKWEED, SPIRODELA POLYRHIZA 


\subsection{Introduction}

Environmental variability presents a challenge for organisms, and research on adaptation to unpredictable environments is a focus of evolutionary biologists. Natural selection, in the presence of heritable phenotypic variation, is expected to result in optimal trait values with respect to a particular environment. However, in variable environments the optimal trait value may continuously shift. Persistence over the longer term has required survival and reproduction not only under expected or 'average' conditions, but under a range of fluctuating conditions (Simons 2014; Furness et al. 2015).

There are several evolutionary mechanisms that could influence persistence in variable environments. Which adaptive strategy evolves will depend upon the environmental parameter space that an organism finds itself in (Simons 2014; Botero et al. 2015; Proulx and Teotónio 2017); specifically, on the level of environmental unpredictability and the timescale of fluctuation (Botero et al. 2015). Strategies include conservative bet hedging, diversification bet hedging, phenotypic plasticity, or adaptive tracking (Simons 2011; Botero et al. 2015).

In environments where variable conditions are predictable, adaptive phenotypic plasticity may evolve, in which phenotypic expression is shifted towards an optimum value along a norm of reaction in direct response to an environmental cue (Bradshaw 1965; Via and Lande 1985; Scheiner 1993; Ghalambor et al. 2007; Simons 2014; Furness et al. 2015). Although there is abundant evidence for adaptive phenotypic plasticity in nature (Via and Lande 1985; Price et al. 2003; Ehrenreich and Pfennig 2016; Li et al. 2016), it cannot evolve when the optimal phenotypic response to changed environments is unpredictable (Maxwell and Magwene 2017).

Diversification bet hedging is a risk aversion strategy wherein individuals produce a variety of phenotypes (e.g. offspring) such that at least a subset will exhibit nonzero reproductive success when the optimal phenotype is unpredictable, thus reducing fitness variance among generations (Dempster 1955; Cohen 1966; Seger and Brockmann 1987; Simons 2009; Martínez-Ruiz and García-Roger 2015). Bet 
hedging maximizes the geometric-mean fitness (the $n$th root of the product of $n$ fitness values) over the longer term rather than expected fitness each generation, and results in traits that appear to be maladapted over the short-term (Cohen 1966; Seger and Brockmann 1987; Simons 2011; Graham et al. 2014). Thus, the geometric-mean fitness represents the long-term fitness of a genotype or lineage under fluctuating environmental conditions (Seger and Brockmann 1987; Simons 2011; Martínez-Ruiz and García-Roger 2015). It is difficult to test bet hedging theory because fitness must be established through a sequence of environments over a long time frame (Simons 2011).

Spirodela polyrhiza (Araceae), or Greater Duckweed, is an appropriate organism for use in the study of bet hedging traits for several reasons. This species produces an overwintering resting "turion" stage that, like dormant seeds, allows persistence through otherwise fatal conditions. Furthermore, its small size and high growth rate makes long-term and simultaneous experimental trials feasible. $S$. polyrhiza is a floating, iteroparous angiosperm in the subfamily Lemnoideae which has shown neotenous reduction from other members of the Araceae family (Wang et al. 2014). It typically reproduces asexually through vegetative budding of daughter fronds from two meristematic pockets (Jacobs 1947), and under optimal conditions S. polyrhiza has a biomass doubling time of approximately 28 hours (Kutschera and Niklas 2015). S. polyrhiza also produces "turions", which, like regular fronds, bud from the mother's meristematic pockets (Jacobs 1947; Appenroth 2002a). Turions are denser and more starch-rich than regular fronds, and thus sink to the sediment surface where they enter a dormant state (Oláh et al. 2015; Mejbel and Simons 2018). Although turions share many phenological parallels to seeds, turions are asexually-produced vegetative organs (Appenroth et al. 1996). S. polyrhiza is one of a number of duckweed species that form turions in response to cues for approaching unfavorable environmental conditions (Appenroth 2003). Unfavorable conditions in this case could mean limited nutrient levels (Appenroth et al. 1989, 1996; Caicedo et al. 2000), cooler temperatures (Jacobs 1947; 
Perry 1968; Oláh et al. 2017) such as fall conditions, or the presence of heavy metals or other contaminants (Oláh et al. 2014, 2015).

Once favorable environmental conditions return, turions reactivate in response to cues that remain largely unknown, and produce and trap a $\mathrm{CO}_{2}$ bubble that buoys them to the surface where they resume growth (Jacobs 1947; Oláh et al. 2017).

Prior work on S. polyrhiza suggests that turion phenology may act as bet hedging in seasonal environments. In a field study, Compton (2013) monitored turion phenology throughout the year in Elgin, Ontario using floating traps and found that both reactivation and formation timing varies within and among natural ponds. Turion reactivation was found to occur from May 18 to November 7 (a 173day range). Similarly, turion formation was found to occur within the same ponds between July 26 and November 7 (a 104-day range). This high variability in the timing of these life-history events suggests the potential existence of a diversification bet hedging strategy (Compton 2013). However, an observational field study cannot determine whether the source of this extreme variability can be accounted for by genetic differences, spatial-environmental heterogeneity, or perhaps a correlation between formation and reactivation times with the potential for multi-year dormancy. Laboratory work has since found that variability in the timing of turion formation is generated by birth order among genetically identical individuals, and may thus act as a diversification strategy: for a given temperature, the probability that a mother frond will produce a turion rather than a regular frond increases with the mothers' birth order (Mejbel and Simons 2018). The source of variability in the timing of reactivation observed during the spring and summer in the field, however, remains unknown.

Immediately following spring ice melt in temperate climates, water surface is a fully untapped niche space. However, early spring growth is not a simple 'race' to occupy habitat for S. polyrhiza, because there is a risk of turion reactivation during an early warm period that is followed unpredictably by re-freezing (Kramer 1994; Rigby and Porporato 2008; Hufkens et al. 2012) of surface waters that 
would kill any reactivated turions (Adamec 2004). Such a trade off between the competitive advantage of early reactivation and an unpredictable re-freezing of the water's surface in the early spring is expected to result in diversification bet hedging.

S. polyrhiza is effectively a clonal organism, and it is not known how variation in turion reactivation might be generated among genetically identical individuals. Following turion formation, dormancy breaks from exposure to cold conditions; however, reactivation is supressed until environmental cues are received (Jacobs 1947; Lacor 1969; Ley et al. 1997; Oláh et al. 2015, 2017). Little work has been done on the specific conditions inducing reactivation of S. polyrhiza turions, although it is speculated that photoperiod, light intensity or quality, temperature, and nutrients could play a role in either signalling or inhibiting reactivation (Oláh et al. 2017). If all individuals within clonal lineages of $S$. polyrhiza reacted in the same way to environmental cues, reactivation would be synchronous. Therefore, phenotypic variation within clones must be derived from non-genetic sources.

Transgenerational birth order effects from a frond's mother appear to have a strong influence on phenological behaviour of turion production (Barks and Laird 2016; Mejbel and Simons 2018). The variability in reactivation timing observed within a season suggests that individual response to cues may also differ as a function of birth order. If different phenotypes (birth orders) respond to the environment differently, a phenotype-environment interaction could be the driver of a diversification bet hedging strategy in the timing of turion reactivation.

In this thesis, I present the results of two studies in which I test for the effects of mother and daughter (turion) birth order on reactivation phenology and performance in S. polyrhiza. I hypothesize that variation in turion reactivation occurs within genotypes as a potential diversification bet-hedging strategy. It was predicted that birth order and temperature were the main drivers of phenological variability among genetically identical turions whereby the mother, and to a greater extent, the turion's birth order would impact the order of reactivation and cooler temperatures would draw out the effect. 
To test these predictions, a laboratory study examined (at three different temperatures) birth order effects on variation in turion reactivation timing. Additionally, an outdoor mesocosm study assessed whether turion birth order effects influence growth rates. The mesocosm study included temperature supplementation treatments across temporal trials to mimic effects of within season timing of reactivation.

Birth order effects resulting in phenological variation in spring turion reactivation would be suggestive of diversification bet hedging that reduces risk associated with spring re-freezing events. I also consider that the optimal diversification strategy might depend not only on the production of variation in phenology, but also on the relative performance of turions of different birth orders. Although this work tests for sources of diversification in S. polyrhiza, a quantitative assessment of the optimality (Orzack and Sober 1994; Simons 2011) of bet hedging would require determining the fitness consequences of birth order reactivation phenology over multiple seasons, and is beyond the scope of this study.

\subsection{Materials and Methods}

\subsubsection{Growth and Maintenance of the Population}

To reduce the risk of clone-specific anomalous results, two geographically distinct accessions of $S$. polyrhiza were acquired for this study. One was collected from Queens University Biological Station (Elgin, Ontario: $44^{\circ} 34^{\prime} 01^{\prime \prime} \mathrm{N}$ and $76^{\circ} 19^{\prime} 26^{\prime \prime} \mathrm{W}$ ) by past lab members in 2011 . The second was collected

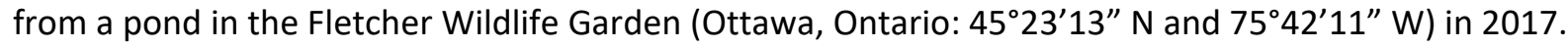
Both populations were initially cultivated in Appenroth's growth medium (Appenroth et al. 1996) by reactivating turions and allowing them to bud. Populations were continuously propagated on a light shelf at $26^{\circ} \mathrm{C}\left( \pm 1.5^{\circ} \mathrm{C}\right)$ with a $14 / 10 \mathrm{hr}$ day/night photoperiod.

Maternal birth-order effects are well known in duckweed species (Barks and Laird 2016;

Ankutowicz and Laird 2018; Mejbel and Simons 2018) and were controlled by obtaining first daughters 
of first daughters (fronds) for at least four generations prior to initiating lab and mesocosm work (Barks and Laird 2015, 2016; Mejbel and Simons 2018). Preparatory work for this study was performed by growing fronds individually in 12-well plates filled with growth medium. These multi-well plates were placed in the centre of a Biochambers SG-30 seed germinator set to constant $21^{\circ} \mathrm{C}$ with a $14 / 10 \mathrm{hr}$ day/night photoperiod.

\subsubsection{Laboratory Study of Turion Reactivation Time}

Turions of different birth orders were needed to assess the timing of reactivation among phenotypes. For this study, combinations of mother and daughter (turion) birth orders 3, 5, 7, and 9 were used. These turions were produced using a thermogradient apparatus with a $\sim 5^{\circ} \mathrm{C}$ temperature range of $10-15^{\circ} \mathrm{C}$, based on prior information on the generation of turions among mother birth orders across temperatures (Mejbel and Simons 2018). Prior to use, the stability of the thermogradient was monitored for two weeks. Floating foam rafts fitted with plastic dividers (to segregate fronds) and mesh screen affixed to their underside (for turion capture) were placed along the thermogradient. A photograph of the thermogradient is provided in Appendix A-1

To generate daughter turions of the four focal birth orders for use in this study, two replicates of the four focal birth order mothers for both genotypes (totalling 16 individuals) were represented at the five temperature positions along the thermogradient. The thermogradient was divided into two separately sealed lanes (with one of the two replicates in each) and the placement of the fronds was randomized at each temperature position within each lane. Upon the formation of a focal daughter turion (birth order $3,5,7$, or 9), the date of formation and its birth order was recorded, and it was photographed (and calibrated) under an Olympus SZX12 microscope with an attached Lumenera Infinity 3 camera for later measurement (ImageJ 1.52a). Collected turions (yield is summarized in Figure 2.1) were stored in dark vessels and after-ripened at $5 \pm 2^{\circ} \mathrm{C}$ for a minimum of two weeks (Jacobs 1947). 

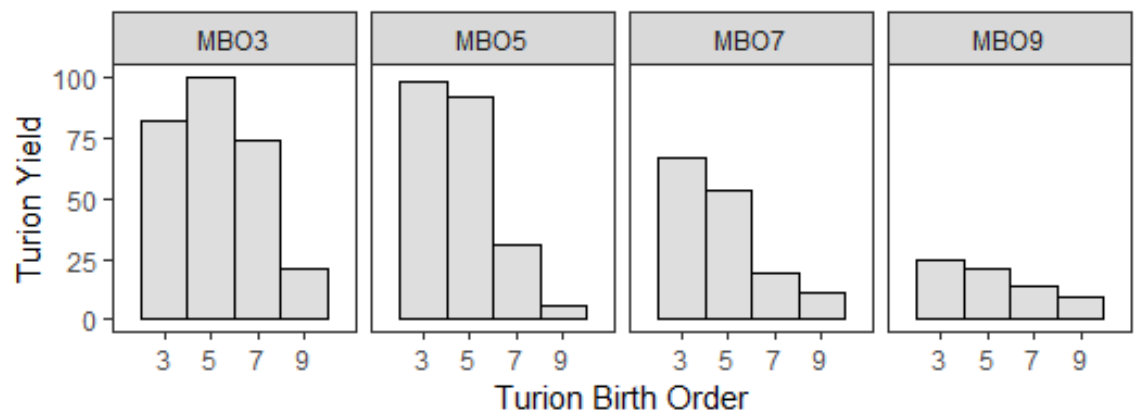

Figure 2.1: Turion yield for reactivation study for combinations of turion birth orders and mother birth orders (MBO).

To observe reactivation, after-ripened turions were transferred to an SG-30 seed germinator, where an array of $20 \mathrm{~mL}$ test tubes filled with medium each contained a single turion which rested on the bottom. Because it is expected that reactivation phenology will be more drawn out at cooler temperatures experienced early in the season than at warmer temperatures, available turions were divided among three sets of conditions for reactivation: $10^{\circ} \mathrm{C}$ and $18^{\circ} \mathrm{C}$ treatments using two SG-30 seed germinators (14/10hr day/night), and a $26^{\circ} \mathrm{C}$ treatment on a light shelf (14/10hr day/night). The placement of test tubes was randomized with respect to birth order in the centre of each shelf and seed germinator. The test tubes were checked every 3-5 hours over a 15 -day period (for the $10^{\circ} \mathrm{C}$ and $18^{\circ} \mathrm{C}$ treatments) and every 6-12 hours over a 4-day period (for the $26^{\circ} \mathrm{C}$ treatment) to track and record the timing of reactivation among the turions; reactivation time was considered to be the time of surfacebreach. A photograph of the test tube array is provided in Appendix A-1.

Statistical analyses were conducted using the statistical software R (v3.5.0). In all cases, mother and daughter (turion) birth orders were treated as categorical factors and ANOVAs were conducted using Type III sums of squares except where noted. The R scripts for both the laboratory reactivation time and mesocosm studies are provided as Appendix B and Appendix $C$.

The prediction that reactivation temperature as well as mother and daughter (turion) birth order influence the timing of turion reactivation was tested using a factorial ANOVA. A post-hoc Tukey test was then conducted to identify differences among levels where significant effects were found. 
It is expected that cold exposure during overwintering would remove pre-winter effects associated with turion formation timing, as it does following stratification in seed germination; however it is possible that the timing of reactivation could be related to timing of turion formation the previous season. An ANCOVA was conducted to test the effects of turion formation timing on the timing of reactivation. The turion collection procedures used in this experiment made elucidating this potential interaction challenging - i.e. early birth orders were in general collected first, where in nature they would be formed more continuously. The ANCOVA thus also included turion birth order along with reactivation temperature and the covariate, timing of turion formation. Given the study procedures (i.e. transfer of turions to cold storage immediately following formation), this analysis assumes that in nature, turions that form and sink to the sediment experience conditions unsuitable for reactivation similar to the cold treatment used in this experiment.

There is a negative relationship between birth order and frond size (Barks and Laird 2016; Mejbel and Simons 2018). To test whether turion size exhibits a similar relationship and whether size alone can predict reactivation time, an ANCOVA including turion size (measured as width, see Mejbel and Simons 2018), birth order, and reactivation temperature was conducted.

\subsubsection{Mesocosm Growth Rate Study}

To assess the environment-dependent effects of turion birth order on performance, an outdoor mesocosm study was conducted. To achieve this, an array of mesocosms was constructed from insulated 100 US Gal. cattle tanks containing floating enclosures (approximately 30 per mesocosm) to house a single turion each. Enclosures were constructed from short segments of 6" diameter tubing fitted with buoyant foam and an underside covered in mesh to prevent the loss of fronds. Two trials, a preliminary trial and a main trial, were performed with starting dates offset by three weeks. Each trial was comprised of nine mesocosms, each subject to one of three temperature treatments with three replicates. Treatment levels included uncontrolled (ambient) mesocosms and mesocosms controlled to 
ambient plus two degrees and ambient plus five degrees. Each mesocosm in the array was equipped with a submerged temperature probe suspended just above the bottom. Those mesocosms that were selected for temperature control (randomized placement) included a 300W Finnex titanium submersible heater located near the bottom of the tank, offset from the temperature probes so as to minimize direct influence. Temperature probes and heaters were connected to a Campbell Scientific datalogger (CR6) and relay controller (SDM-CD16AC) to provide active temperature monitoring and control. Equal amounts of $0.5-0.5-0.5$ fertilizer were added to each mesocosm for nutrient provision. Photographs of the mesocosms and equipment involved in this setup are provided in Appendix A-2.

Environmental chemistry was monitored within each mesocosm using a Hanna Instruments HI98194 handheld multi-metre on an approximately weekly basis to monitor levels and trends in $\mathrm{pH}$, oxidative-reactive potential, dissolved oxygen, electrical conductivity, total dissolved solids, and resistivity. These measurements provide insight into uncontrolled differences among mesocosms that may affect growth rate.

Combinations of mother and daughter (turion) birth orders $(3,5,7$, and 9$)$ were used in the mesocosm study to evaluate performance differences among birth orders and temperatures. Cool temperatures are required for fronds to produce turions, particularly for early birth order mothers to produce early birth order turions (e.g. a third birth order mother producing a third birth order turion). To produce the required assortment of turions, first order mothers were placed on a light shelf at $26^{\circ} \mathrm{C}$ $\left( \pm 1.5^{\circ} \mathrm{C}\right)$ with a photoperiod of $14 / 10$-hr day/nights. The first order mother fronds on the light shelf were allowed to produce daughter fronds within the multi-well plates. Daughter fronds of focal birth orders $(3,5,7$, and 9$)$ were collected and moved into a cool SG-30 seed germinator $\left(12^{\circ} \mathrm{C}\right.$ with $14 / 10-\mathrm{hr}$ day/night photoperiod).

The propensity to produce turions increases with ascending mother birth order and decreasing temperature; thus, later birth order mothers readily produce turions at warmer temperatures - 
between 10 and $18^{\circ} \mathrm{C}$ (Mejbel and Simons 2018). Therefore, temperatures were increased through the course of turion production with increasing daughter turion birth order, thereby also increasing the rate of turion formation. This is important because at temperatures as low as $12^{\circ} \mathrm{C}$, higher birth order turions could not be produced during a mother's short lifespan. Temperatures were increased from $12^{\circ} \mathrm{C}$ to $14^{\circ} \mathrm{C}$ to $16^{\circ} \mathrm{C}$ as turion birth orders increased. Collected turions (yield illustrated in Figure 2.2) were placed individually into dark vessels and after-ripened for a minimum of two weeks to break their innate dormancy (Jacobs 1947).

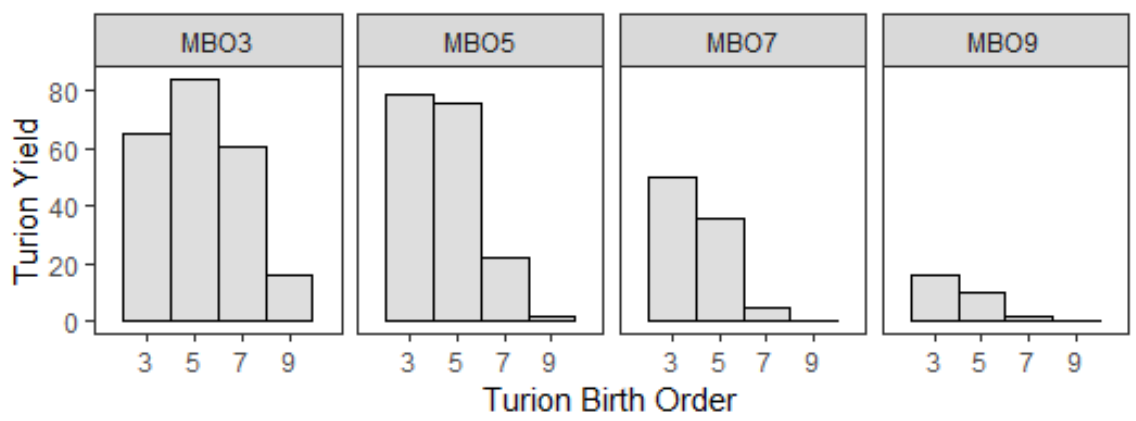

Figure 2.2: Turion yield for growth rate study for combinations of turion birth orders and mother birth orders (MBO).

After-ripened turions were digitally photographed for later measurement in the same manner as in the reactivation study. Turions were then reactivated in preparation for placement into the mesocosms. The goal was to ensure that turions are introduced to the mesocosms at the same time and experience the same environments; reactivation was therefore performed at $26^{\circ} \mathrm{C}\left( \pm 1.5^{\circ} \mathrm{C}\right)$ and $14 / 10-\mathrm{hr}$ day/nights to encourage simultaneous reactivation, as described previously for the reactivation study. Reactivation data from this study were included as the warm $\left(26^{\circ} \mathrm{C}\right)$ temperature treatment in the laboratory reactivation study.

Following a growth period of approximately 1.5 to 2 months, growth within each floating enclosure was evaluated. The number of individual mature fronds was counted and collected for biomass measurement. Populations (from each enclosure) were placed onto pre-dried and weighed weigh paper and were dried in a gravity convection oven (Shel Lab 1370GM) for 36 hours. Dried samples 
were weighed with a fine balance (Mettler Toledo AG285, $\pm 0.01 \mathrm{mg}$ ) and growth rates based on frond number as well as dry biomass were calculated.

Growth rate $(r)$ was calculated using,

$$
r=\frac{\ln \left(n_{t}\right)-\ln \left(n_{o}\right)}{t}
$$

where $n_{t}$ and $n_{o}$ are the number of individual fronds at time $t$ and time zero, respectively. Each population began as a single turion; therefore, $\mathrm{n}_{\mathrm{o}}=1$ in each instance. This same method was used to calculate growth rate from dry biomass, where an assumed initial dry biomass of $0.0001 \mathrm{~g}$ was used to represent the mass of a single turion. Growth rates based on count and dry biomass are correlated $\left(r^{2}\right.$ Ambient $\left.=0.387, r^{2}{ }_{\text {Ambient }+2^{\circ} \mathrm{C}}=0.668, r^{2}{ }_{\text {Ambient }+5^{\circ} \mathrm{C}}=0.859\right)$. Therefore, only the dry biomass analysis results are presented; however, all analyses were conducted using both response variables and any qualitative differences in results reported. Of note is increased standard error (SE) at lower temperature treatments $\left(\mathrm{SE}_{\mathrm{Ambient}}=0.086, \mathrm{SE}_{\mathrm{Ambient}+2^{\circ} \mathrm{C}}=0.051, \mathrm{SE}_{\mathrm{Ambient}+5^{\circ} \mathrm{C}}=0.043\right)$; this could be due to possible increased measurement error with lower frond counts per sample compared to the warmer mesocosms.

Due to limited turion yield in the higher birth orders, a factorial design could only be achieved among mother and daughter (turion) birth orders 3 and 5, which were included in all mesocosms with replication (referred to as the "core" factorial design). The remainder of the generated turions (including those of higher birth order) were allocated equally among the mesocosms to maximize birth order representation and replication among temperature treatments. The preliminary trial was used to corroborate the fuller analyses from the main trial. Data loss was experienced in both trials of the experiment, most notably in the preliminary trial (representing $85 \%$ of the combined data loss) where turions were lost through either death or by escaping from their enclosures (an issue that was resolved for the main trial). Additionally, a loss of a portion of enclosure labels in the preliminary trial disrupted tracking capabilities for the affected turions. The main trial experienced little data loss through turion death only and equal representation within the "core" factorial component of this study was retained. 
Results for birth order 7 and 9 turions were included in secondary analyses to examine qualitative consistency among the wider results of the study. Furthermore, preliminary trial results were not used in the main analysis but were used for complimentary analyses and post-hoc comparison.

An ANOVA was performed to test for the influence of temperature treatment, mother and daughter (turion) birth orders, their interactions, and the random nested effect of mesocosm within treatment, on lineage performance. This model was created using the Linear Mixed-Effects Model (Ime4) package for R.

Similar to the reactivation study, an ANCOVA was conducted to test for the effect of turion size (as width) on growth rate. This model also included temperature treatment and the random variable, mesocosm nested within treatment.

\subsection{Results}

\subsubsection{Laboratory Study of Turion Reactivation Time}

Overall reactivation time variation was substantial (Figure 2.3), and differed among temperatures $\left(F_{2,650}=588.8, p<2.2 \mathrm{e}-16\right)$.

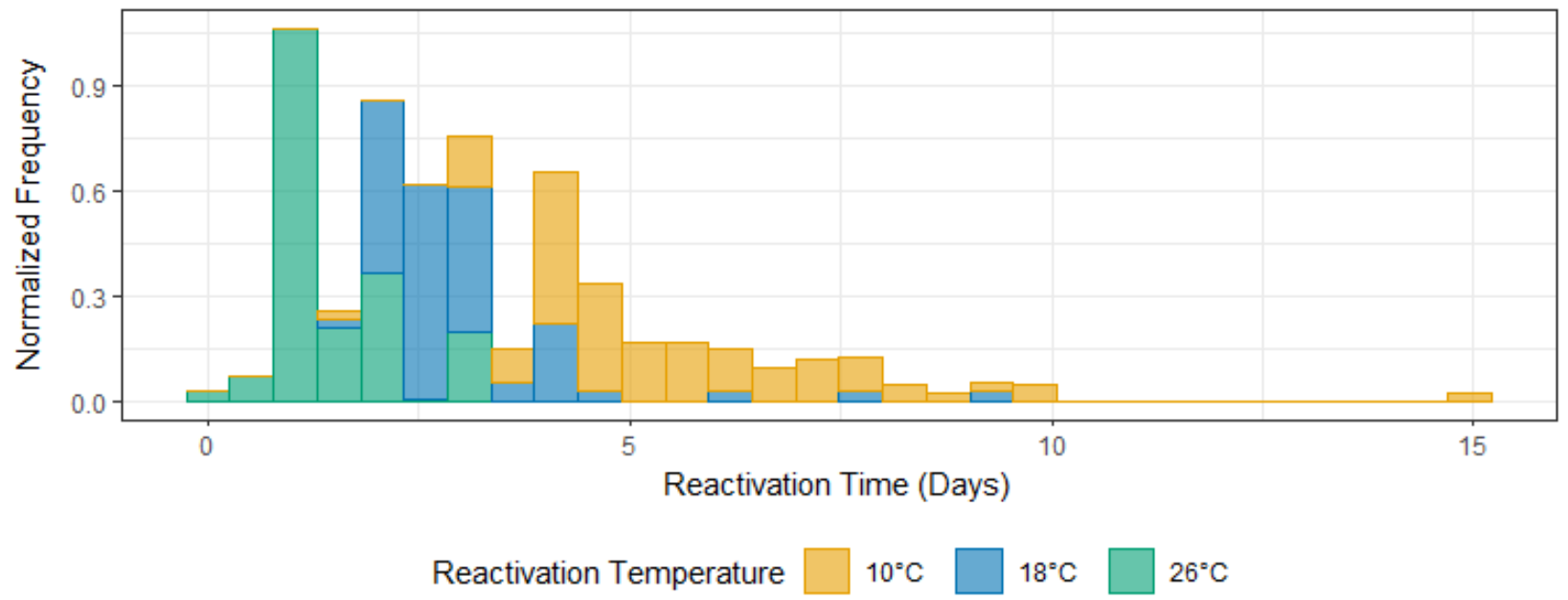

Figure 2.3: Overall reactivation timing of S. polyrhiza turions in the lab for the three temperature treatments (stacked). Normalized frequency values are scaled by dividing the frequency of each bin by the sum of all of the frequencies within a given temperature treatment; these are thus calculated independently for each treatment. 
The full ANOVA including effects of birth order found that all predictor variables had a significant effect on turion reactivation time (Table 2.1). A post-hoc Tukey test identified differences among all reactivation temperatures and turion birth orders, except between turion birth orders 5 and 7 . Although mother birth order was marginally significant according to the ANOVA, the post-hoc analysis failed to identify any levels that differed. Furthermore, treating mother and turion birth order as continuous rather than categorical did not qualitatively alter the results. Early birth order turions tended to reactivate earlier than those of later birth order (Figure 2.4).

Table 2.1: Fully-factorial ANOVA for effects of reactivation temperature (TEMP), mother birth order (MBO) and daughter turion birth order (TBO) on time to turion reactivation in S. polyrhiza.

\begin{tabular}{lllrl}
\hline Source & df & SS & \multicolumn{1}{c}{ F-ratio } & P \\
\hline TEMP & 2 & 685868 & 850.241 & $<0.001^{*}$ \\
MBO & 3 & 3311 & 2.736 & $0.0428^{*}$ \\
TBO & 3 & 36651 & 30.290 & $<0.001^{*}$ \\
MBO:TBO & 9 & 14093 & 3.882 & $<0.001^{*}$ \\
TEMP:MBO & 6 & 17394 & 7.187 & $<0.001^{*}$ \\
TEMP:TBO & 6 & 34433 & 14.228 & $<0.001^{*}$ \\
TEMP:MBO:TBO & 16 & 27870 & 4.319 & $<0.001^{*}$ \\
\hline
\end{tabular}



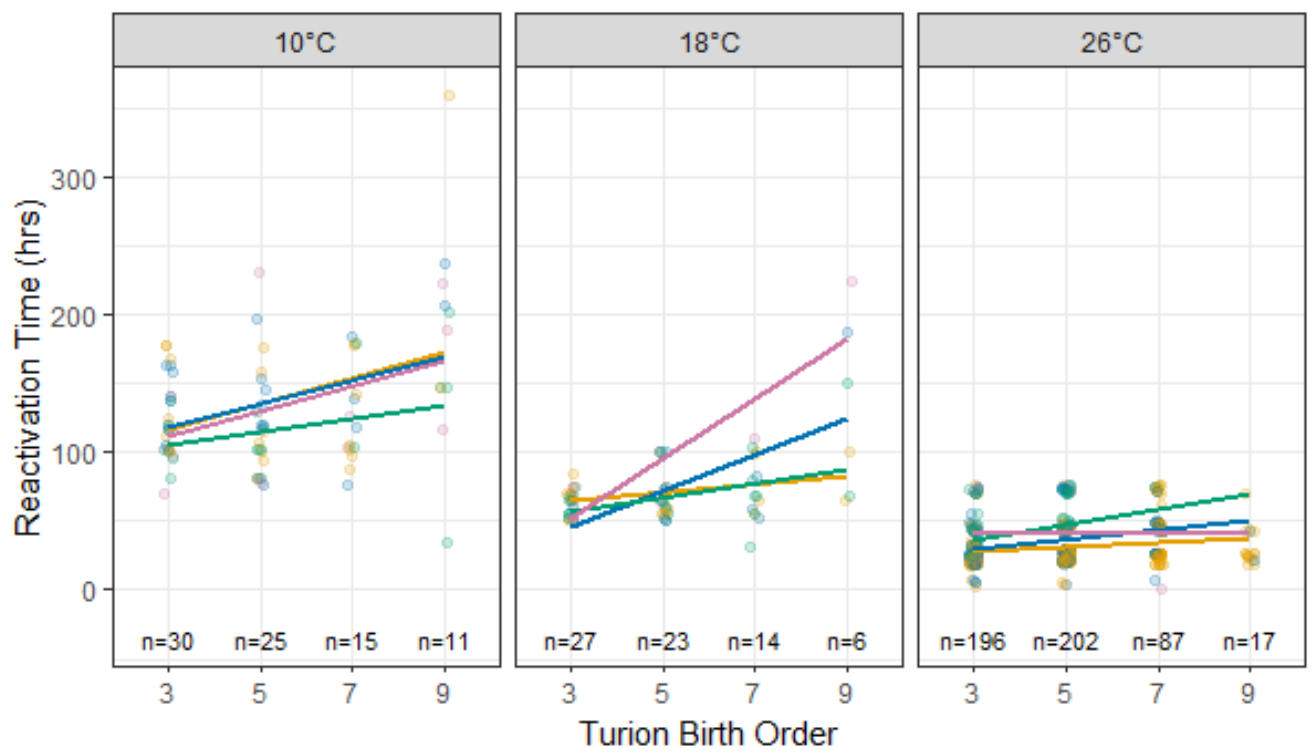

Mother Birth Order $-3-5-7 \div 9$

Figure 2.4: Reactivation time of S. polyrhiza turions across turion and mother birth orders for the three temperature treatments. Effect of turion birth order is fitted separately for each mother birth order. Sample size $(n)$ of each turion birth order group noted at each $\mathrm{x}$-axis tick. Regression lines included for illustration purposes, however, birth order was treated as categorical for the analysis.

Secondary analyses were performed to better inform the interpretation of the main results presented previously. The ANCOVA to test whether the timing of turion reactivation is directly affected by the phenology of formation from the preceding season found a significant effect $\left(F_{1,628}=13.7, P<\right.$ 0.001), though non-directional. Furthermore, the results of a second ANCOVA to test for turion size effects showed a significant negative relationship between size and reactivation time $\left(F_{1,635}=72.3, P<\right.$ 0.001 ), while turion birth order was significant when added to this analysis (size: $F_{1,632}=45.8, P<0.001$ and TBO: $\left.F_{1,632}=21.2, P<0.001\right)$ even when turion size was accounted for.

\subsubsection{Mesocosm Growth Rate Study}

The mixed-effects ANOVA for growth rate showed no significant effects of any of the predictor variables including temperature treatment and birth order (Table 2.2). No qualitative differences were found in further analyses using growth rate based on frond count, using the entire main trial data set 
(i.e. including mother and turion birth orders 7 and 9), the full preliminary trial data set, or both trials' full data sets.

Table 2.2: ANOVA for frond growth rate in S. polyrhiza measured as change in dry biomass. Factors included temperature treatment (TEMP), mother (MBO) and daughter turion birth order (TBO), their interactions, and a random mesocosm effect (MESO) nested within temperature treatment.

\begin{tabular}{lllll}
\hline Source & df & SS & F-ratio & $\mathbf{p}$ \\
\hline TEMP & 2 & $5.90 \mathrm{e}-05$ & 0.3315 & 0.7300 \\
MBO & 1 & $2.50 \mathrm{e}-05$ & 0.2814 & 0.5970 \\
TBO & 1 & $2.74 \mathrm{e}-06$ & 0.0308 & 0.8611 \\
TEMP:MBO & 2 & $1.11 \mathrm{e}-04$ & 1.2430 & 0.2931 \\
TEMP:TBO & 2 & $6.62 \mathrm{e}-05$ & 0.7441 & 0.4779 \\
MBO:TBO & 1 & $2.40 \mathrm{e}-05$ & 0.2695 & 0.6048 \\
TEMP:MBO:TBO & 2 & $3.44 \mathrm{e}-05$ & 0.3866 & 0.6804 \\
MESO(TEMP) & 1 & N/A & N/A & $<0.001 *$ \\
\hline
\end{tabular}

The full model that included birth orders and the random nested mesocosm factor may mask an underlying overall effect of temperature, because when a large proportion of variance is explained by a nested factor, the power to detect real differences in a treatment is reduced (Konstantopoulos 2006, 2008; Hedges and Hedberg 2007). Therefore, an exploratory, post-hoc one-way ANOVA for treatment as a predictor for growth rate was performed and revealed a highly significant effect regardless of whether the two focal birth orders $\left(F_{2,117}=20.3, P<0.001\right)$, or all birth orders were included $\left(F_{2,216}=23.1, P<\right.$ 0.001). Although ignoring the nested factor may produce erroneous results, it should be noted that mean growth rates fell in the predicted order, with ambient temperatures resulting in significantly lower growth rates than either heated treatment by a post-hoc Tukey HSD test, although the $+2^{\circ} \mathrm{C}$ and $+5^{\circ} \mathrm{C}$ levels did not differ significantly. Results for growth rate based on frond count for all data in the main trial revealed a stronger effect of temperature $\left(F_{2,217}=42.4, P<0.001\right)$, with the Tukey HSD test revealing differences among all three levels in the predicted direction (Figure 2.5).

The ANCOVA for growth rate as a function of turion size was shown to be significant for the two focal birth orders $(F=14.3, P<0.001)$, as well as for all birth orders of the main trial $(F=16.0, P<0.001)$. 


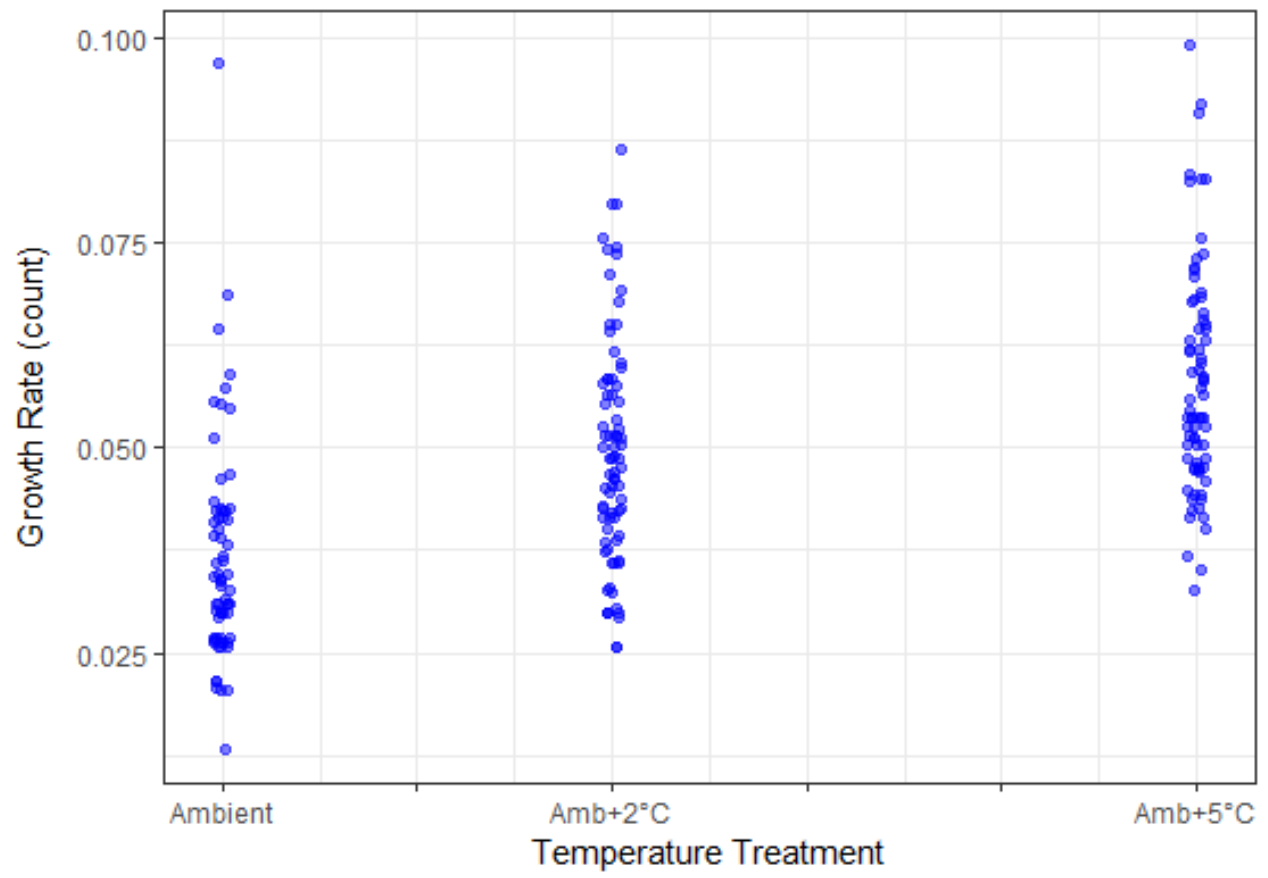

Figure 2.5: Growth rate of S. polyrhiza as a function of temperature treatment. For presentation purposes, treatment is illustrated on an interval scale, although it was treated as categorical for the analyses. Growth rate based on count is used because it better illustrates the differences along temperature treatments.

\subsection{Discussion}

Adaptive phenotypic plasticity and bet hedging are expected to jointly evolve when environmental fluctuations have deterministic and stochastic components (Simons 2014). Variability in the timing of reactivation in S. polyrhiza turions is proposed here as diversification bet hedging around a norm of reaction in response to unpredictability in cyclical seasonal variation. Specifically, I asked whether birth order was responsible for the production of variation in turion reactivation time around a norm of reaction for temperature, and whether birth order contributes to performance differences in clonal populations of S. polyrhiza. An observation that birth order generates variation in timing events around a thermal norm of reaction would provide evidence that phenotype-environment interaction is a potential driver of diversification bet hedging in this species. 
Variation in the timing of reactivation observed among turions within clones was related to birth order, with earlier birth orders reactivating sooner within a given thermal environment. Furthermore, interactions with temperature were found to scale the magnitude of this turion birth order effect: the birth order effects increased with decreasing temperatures. As expected, the effect of a turion's mother birth order is weaker than that of its own birth order. The analysis for turion formation time as a predictor for reactivation time found a significant relationship suggesting that the length of the overwintering period could be relevant; however, it is possible that turion collection procedures (birth orders collected in order) have some influence over this result. To explore this, the same analysis was performed using only daughter birth order 3 turions to ask whether removing turion birth order effects from the analysis would alter the outcome. A marginally non-significant relationship was found $\left(F_{1,236}=\right.$ 3.571, $P=0.06$ ) suggesting that it is possible that, with seed germination, a cold after-ripening treatment synchronizes the turions leading into the spring which eliminates the influence of pre-winter timing effects. Due to the reduced power of this exploratory analysis, more work could be done to elucidate these effects. This analysis makes the assumption that formed turions enter cold treatment-like conditions when they sink to the sediment surface regardless of the time of year they are formed. The result must be interpreted from the perspective that temperature and light exposure at the sediment surface would vary among water bodies depending on physical and chemical properties of the water feature and this assumption may not always hold. Furthermore, due to the procedures used for the production of turions for the two studies, I was unable to fully dismiss turion formation conditions (including temperature) as a possible contributor to the observed birth order effects.

There is a known negative relationship between birth order and frond size in duckweed (Barks and Laird 2016; Mejbel and Simons 2018). Size is thus a characteristic of birth order; however, the effect of turion birth order on reactivation time remains significant even after accounting for turion size (measured as width; see Mejbel and Simons 2018) in an ANCOVA. The two genotypes used in this study 
differed in reactivation time $(p<0.001)$, the Ottawa genotype tended to reactivate earlier than the Elgin genotype, but showed no qualitative differences in trends in within-individual variation in reactivation time which was the focus of this study. See Appendix E for an extended analysis of genotype effects on reactivation time.

The results of this study show significant variability in reactivation time among clones given the stable laboratory conditions. The range in reactivation time shown here, however, is small in comparison to the temporal variability observed in natural ponds by Compton (2013). Under natural conditions, turions will overwinter at varying depths and position within ponds, affecting light intensity, temperature, and other biotic and abiotic conditions. It is therefore expected that the temporal variability in reactivation time would be greater than in a controlled environment. The differences in turion reactivation between our studies may also be attributed to within-pond genotypic differences or possible multi-year or within-season dormancy cycles.

The effects of turion birth order and temperature on the timing of reactivation are expected to have implications for survival and performance under variable environments. The early birth order turions that reactivate early in the spring would experience little competition but would also face a high risk of mortality in the event of pond re-freezing. Alternatively, late birth order, late-rising turions would experience lower risk and more favourable growing conditions but would face increased competition and a reduced growing season relative to those that reactivated early. This is especially true in natural environments where a more drawn out reactivation distribution is expected. A similar trade-off structure is exhibited for seed germination; for example, Mediterranean woody plant species have also been shown to display variability in germination timing to overcome a trade-off between early frost risk and season length and competition (Castro-díez et al. 2012).

Inherent growth rate differences among birth orders under a particular thermal environment would modify the fitness consequences of reactivation phenology; however, the mesocosm study's 
failure to detect such differences suggests that diversification within clones is generated by birth order phenology alone. Alternatively, the lack of observed performance differences may be a product of study limitations.

\subsubsection{Study Limitations}

The observation of performance differences among birth orders under laboratory conditions in other studies (Barks and Laird 2016; Mejbel and Simons 2018), and the observation in the present mesocosm study that turion size affected growth rate $(p<0.001)$ raises the possibility that temperaturespecific performance differences among birth orders could exist but were not detected in this study. For illustration purposes, Figure 2.6 presents the expected effect that turion birth order would have on lineage growth rates. There are several possible reasons why this effect was not observed.

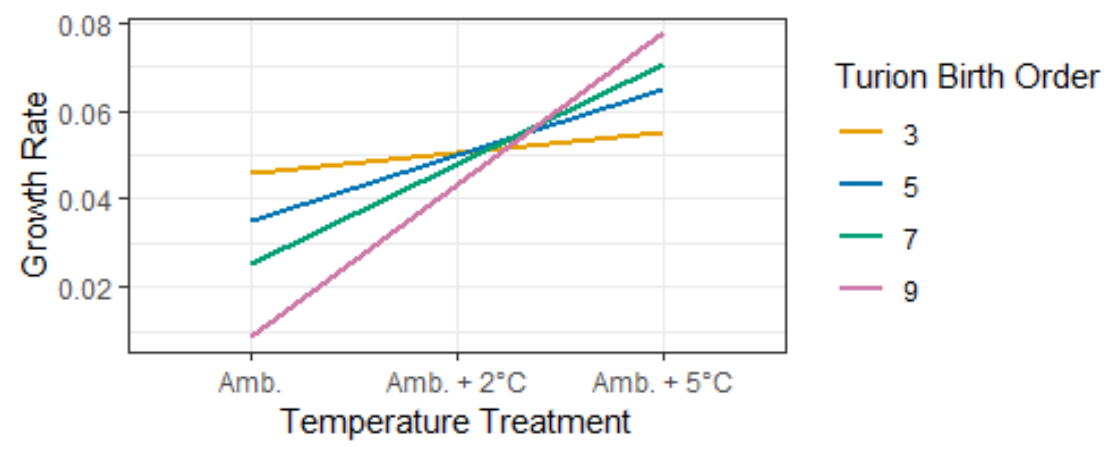

Figure 2.6: Predicted growth rate response to turion birth order and treatment effect. This graph was based on a hypothetical dataset to present the expected results of this study. For simplicity, mother birth order was excluded.

First, the mesocosm study was uncontrolled in that it was susceptible to natural variation in ambient temperatures. The period over which the study was conducted lacked the full range of temperatures characteristic of a natural growing season. The laboratory study on reactivation timing suggests that the effect size of birth order is magnified at lower temperatures; the addition of a mesocosm trial during colder periods may have revealed underlying differences in performance among birth orders. Although the ambient mesocosms experienced cool temperatures (minimum pond temperatures were $13.9^{\circ} \mathrm{C}$ and $15.8^{\circ} \mathrm{C}$ for the preliminary and main trails, respectively), low temperature 
exposure was minimal and only experienced at the beginning of the experiment which may not have been strong enough to affect the results.

Second, it is possible that birth order effects are initially strong, but diminish over time as other birth orders are produced by the focal birth orders and by their daughter fronds, etc. On the other hand, duckweed growth is initially exponential (Appenroth et al. 2001; Appenroth 2003), and the initial performance benefits among turions would be expected to magnify as the lineages grow. Growth was based on count (and biomass) at the termination of the trial; more frequent monitoring for biomass is not possible because it is destructive, but it is possible that higher temporal resolution of counts could have revealed birth order effects.

A third factor that could have led to equivocal results in the mesocosm study was low statistical power to detect growth-rate differences. To address this possibility, a power analysis was performed. Using an effect size of 0.25 (Cohen 1988; using 'Cohen's d' effect size equation, an effect size of 0.28 was achieved between turion birth orders 3 and 5 for mother birth order 3), an alpha significance level of 0.05, a power of 0.8 (Maccallum et al. 1996; Hedges and Hedberg 2007), and 48 groups (three temperature treatments, four mother birth orders, and four turion birth orders), the power analysis revealed a sample size requirement of 11 samples per group. The target sample size per group in the "core" factorial experiment was 12 to 14 ; however, due to some data loss in the second trial (see Materials and Methods), only 6 of 12 groups in the "core" experiment achieved this requirement with the lowest sample size being 8 individuals per group. Reduced power may have influenced the results; however, sample sizes were within the correct range suggesting that it is likely not the only contributing factor.

Finally, there were substantial differences in uncontrolled but measured conditions other than temperature among mesocosms, potentially obscuring birth order effects on performance generated by temperature. Two-way ANCOVAs including both preliminary and main data sets to assess growth rate 
(dry biomass) as a function of temperature treatment and (1) $\mathrm{pH}\left(\mathrm{F}_{1,283}=12.9, P<0.001\right),(2)$ oxygenreduction potential $\left(F_{1,283}=42.4, P=0.160\right),(3)$ dissolved oxygen $\left(F_{1,283}=3.5, P=0.063\right),(4)$ total dissolved solids $\left(F_{1,283}=56.1, P<0.001\right)$, (5) electrical conductivity $\left(F_{1,283}=61.6, P<0.001\right)$, and (6) resistivity $\left(F_{1,283}=8.7, P=0.003\right)$ finds that $\mathrm{pH}$, total dissolved solids, electrical conductivity, and resistivity likely contributed to the high variability in results among mesocosms.

\subsubsection{Future Direction}

The results of this study provide a foundation for further investigations into the life-history patterns of S. polyrhiza and its proposed bet hedging traits. Evidence for the existence of bet hedging would require demonstrating that geometric-mean fitness is maximized at the cost of average or generational expected fitness (Simons 2011). This could be achieved through a simulation model, which could be used to compare the performance of a candidate bet hedging population with an alternative non-bet hedging population. The model would need to simulate the growth of multiple overlapping birth orders and generations among many years with unpredictably variable conditions. Valuable to this model, the results of the laboratory reactivation and mesocosm studies provide information with respect to reactivation time and growth rate responses with temperature to support the model's environmental interactions. This would first require further mesocosm study to include lower temperature treatments to complete our basic understanding of growth responses with temperature. Like any test of optimality (Orzack and Sober 1994), a strong test of a bet hedging adaptation would require demonstrating that the bet-hedging trait maximizes long-term fitness given the observed extent of environmental variation (Simons 2011).

The inclusion of turion size in addition to birth order (which appear to be closely linked to one another and to reactivation time) in this model would allow for an investigation into whether turion size represents the parental investment to each birth order. Differential parental investment among birth orders and thus with reactivation time could correlate with environmental risk exposure in the spring as 
a function of offspring survival or success following reactivation. The interpretation of the results of the laboratory reactivation and mesocosm studies (i.e. early birth orders reactivating early relative to late turion birth orders where they would experience cooler conditions and slower growth) could be further explored through this model. It is currently hypothesized that early birth order turions are more "hardy" and have potentially elevated ability to cope with cold stress brought on by early spring conditions. Under this scenario, their probability of surviving cold weather events would need to be such that it is more beneficial than the disadvantages associated with late reactivation.

Additionally, different populations of S. polyrhiza that are exposed to distinct environmental regimes are expected to have evolved different life-history patterns, including the phenology of turion formation and reactivation. S. polyrhiza has a world-wide distribution, and specific predictions about such population differentiation could be generated and tested over a wide geographical area.

\subsubsection{Conclusion}

These studies provided important conclusions related to phenology in the duckweed, S. polyrhiza. The joint influence of temperature and birth order as drivers of variability in turion reactivation timing and evidence towards a positive correlation between growth rate and temperature both provide weight to theories surrounding the ability of clone species such as S. polyrhiza to adapt under unpredictable conditions. This study found differences in cue interpretation among phenotypically distinct turions (birth orders), generating timing differences in turion reactivation. If this variable phenotypic expression presents long-term fitness benefits (geometric-mean fitness), it could be an example of diversification bet hedging to overcome challenges associated with environmental unpredictability. Diversification bet hedging could be the driver of this phenotypic variability and may provide an evolutionary explanation for its existence within populations all over the world. 


\section{References}

Adamec, L. 2004. Ecophysiological characterization of dormancy states in turions of the aquatic carnivorous plant Aldrovanda vesiculosa.

Ankutowicz, E. J., and R. A. Laird. 2018. Offspring of older parents are smaller-but no less bilaterally symmetrical-than offspring of younger parents in the aquatic plant Lemna turionifera. Ecology and Evolution 8:679-687.

Appenroth, K., M. Bischoff, and H. Gabryś. 2000. Kinetics of chromium (V) formation and reduction in fronds of the duckweed Spirodela polyrhiza-a low frequency EPR study. Journal of Inorganic ... 78:235-242.

Appenroth, K., W. Hertel, F. Jungnickel, and H. Augstenn. 1989. Influence of Nutrient Deficiency and Light on Turion Formation in Spirodela polyrhizas (L.) SCHLEIDEN. Biochemie und Physiologie der Pflanzen 184:395-403.

Appenroth, K. J. 2002a. Clonal differences in the formation of turions are independent of the specific turion-inducing signal in Spirodela polyrhiza (great duckweed). Plant Biology 4:688-693.

Appenroth, K. J. 2002b. Co-action of temperature and phosphate in inducing turion formation in Spirodela polyrhiza (Great duckweed). Plant, Cell and Environment 25:1079-1085.

Appenroth, K. J. 2003. No photoperiodoc control of the formation of turions in eight clones of Spirodela polyrhiza. Journal of Plant Physiology 160:1329-1334.

Appenroth, K. J., K. S. Sree, V. Böhm, S. Hammann, W. Vetter, M. Leiterer, and G. Jahreis. 2017. Nutritional value of duckweeds (Lemnaceae) as human food. Food Chemistry 217:266-273. Elsevier Ltd.

Appenroth, K. J., J. Stöckel, A. Srivastava, and R. J. Strasser. 2001. Multiple effects of chromate on the photosynthetic apparatus of Spirodela polyrhiza as probed by OJIP chlorophyll a fluorescence measurements. Environmental Pollution 115:49-64.

Appenroth, K., S. Teller, and M. Horn. 1996. Photophysiology of turion formation and germination in Spirodela polyrhiza. 38:95-106.

Archambault, J. M., W. G. Cope, and T. J. Kwak. 2018. Chasing a changing climate: Reproductive and dispersal traits predict how sessile species respond to global warming. Diversity and Distributions 24:880-891.

Barks, P. M., and R. A. Laird. 2016. A multigenerational effect of parental age on offspring size but not fitness in common duckweed (Lemna minor). Journal of Evolutionary Biology 29:748-756.

Barks, P. M., and R. A. Laird. 2015. Senescence in duckweed: Age-related declines in survival, reproduction and offspring quality. Functional Ecology 29:540-548.

Barrett, R. D. H., and D. Schluter. 2008. Adaptation from standing genetic variation. Trends in Ecology 
and Evolution 23:38-44.

Bell, G., and S. Collins. 2008. Adaptation, extinction and global change. Evolutionary Applications 1:3-16.

Bonnet, T., and E. Postma. 2018. Fluctuating selection and its (elusive) evolutionary consequences in a wild rodent population. Journal of Evolutionary Biology 31:572-586.

Botero, C. A., F. J. Weissing, J. Wright, and D. R. Rubenstein. 2015. Evolutionary tipping points in the capacity to adapt to environmental change. Proceedings of the National Academy of Sciences 112:184-189.

Bradshaw, A. D. 1965. Evolutionary significance of phenotypic plasticity in plants. Advances in Genetics 13:115-155.

Brewer, W. L., C. L. Lippitt, C. D. Lippitt, and M. E. Litvak. 2017. Assessing drought-induced change in a piñon-juniper woodland with landsat: A multiple endmember spectral mixture analysis approach. International Journal of Remote Sensing 38:4156-4176. Taylor \& Francis.

Caicedo, J. R., N. P. Van Der Steen, O. Arce, and H. J. Gijzen. 2000. Effect of total ammonia nitrogen concentration and $\mathrm{pH}$ on growth rates of duckweed (Spirodela polyrrhiza). Water Research 34:3829-3835.

Carter, S. K., D. Saenz, and V. H. W. Rudolf. 2018. Shifts in phenological distributions reshape interaction potential in natural communities. Ecology Letters 21:1143-1151.

Castro-díez, A. P., G. Montserrat-martí, J. H. C. Cornelissen, and S. P. Ecology. 2012. Trade-Offs between Phenology, Relative Growth Rate, Life Form and Seed Mass among 22 Mediterranean Woody Species. Plant Ecology 166:117-129.

Chen, W., L. Peng, L. Jiang, D. A. Pike, C. R. Friesen, and G. Brown. 2018. High altitude frogs (Rana kukonoris) adopt a diversified bethedging strategy in the face of environmental unpredictability. Asian Herpetological Research 9:43-49.

Childs, D. Z., C. J. E. Metcalf, and M. Rees. 2010. Evolutionary bet-hedging in the real world: Empirical evidence and challenges revealed by plants. Proceedings of the Royal Society B: Biological Sciences 277:3055-3064.

Christie, M. R., and C. L. Searle. 2018. Evolutionary rescue in a host-pathogen system results in coexistence not clearance. Evolutionary Applications 11:681-693.

Cohen, D. 1966. Optimizing reproduction in a randomly varying environment. Journal of Theoretical Biology 12:119-129.

Cohen, J. 1988. Statistical Power Analysis for the Behavioral Sciences. 2nd Editio. Lawrence Erlbaum, Hillsdale, N.J.

Compton, M. 2013. The Phenology of Turion Formation in Spirodela polyrhiza as an Adaptation to Unpredictable Environments: Implications for the Evolution of Bet Hedging and Phenotypic Plasticity. Carleton University M.Sc. Thesis. 
Crowley, P. H., S. M. Ehlman, E. Korn, and A. Sih. 2016. Dealing with stochastic environmental variation in space and time: bet hedging by generalist, specialist, and diversified strategies. Theoretical Ecology 9:149-161. Theoretical Ecology.

Dempster, E. R. 1955. Maintenance of genetic heterogeneity. Cold Spring Harbor Symposia on Quantitative Biology 20:25-32.

Edwards, M., A. J. Richardson, and Martin Edwards \& Anthony J. Richardson. 2004. Impact of climate change on marine pelagic phenology and trophic mismatch. Nature 430:881-884.

Ehrenreich, I. M., and D. W. Pfennig. 2016. Genetic assimilation: A review of its potential proximate causes and evolutionary consequences. Annals of Botany 117:769-779.

Franks, S., S. Sim, and A. Weis. 2007. Rapid evolution of flowering time by an annual plant in response to a climate fluctuation. Proceedings of the National Academy of Sciences of the United States of America 104:1278-1282.

Furness, A. I., K. Lee, and D. N. Reznick. 2015. Adaptation in a variable environment: Phenotypic plasticity and bet-hedging during egg diapause and hatching in an annual killifish. Evolution 69:1461-1475.

Geng, Q., T. Li, P. Li, X. Wang, W. Chu, Y. Ma, H. Ma, and H. Ni. 2018. The accumulation, transformation, and effects of quinestrol in duckweed (Spirodela polyrhiza L.). Science of the Total Environment 634:1034-1041. Elsevier B.V.

Ghalambor, C. K., J. K. McKay, S. P. Carroll, and D. N. Reznick. 2007. Adaptive versus non-adaptive phenotypic plasticity and the potential for contemporary adaptation in new environments. Functional Ecology 21:394-407.

Gönen, Ç. 2018. Enhancing Bioconversion Potential of Duckweed by Acid and Hydrogen Peroxide Pretreatment Methods to Improve Biofuel Productivity. Sugar Tech 20:474-481.

Graham, J. K., M. L. Smith, and A. M. Simons. 2014. Experimental evolution of bet hedging under manipulated environmental uncertainty in Neurospora crassa Experimental evolution of bet hedging under manipulated environmental uncertainty in Neurospora crassa. Proceedings of the Royal Society B-Biological Sciences 281.

Hedges, L. V., and E. C. Hedberg. 2007. Intraclass Correlations for Planning Group Randomized Experiments in Rural Education. Journal of Research in Rural Education 22:1-15.

Hufkens, K., M. A. Friedl, T. F. Keenan, O. Sonnentag, A. Bailey, J. O’Keefe, and A. D. Richardson. 2012. Ecological impacts of a widespread frost event following early spring leaf-out. Global Change Biology 18:2365-2377.

Jacobs, D. L. 1947. An Ecological Life-History of Spirodela Polyrhiza (Greater Duckweed) with Emphasis on the Turion Phase. Ecological Monographs 17:437-469.

Kaur, M., M. Kumar, S. Sachdeva, and S. K. Puri. 2018. Aquatic weeds as the next generation feedstock for sustainable bioenergy production. Bioresource Technology 251:390-402. Elsevier. 
Konstantopoulos, S. 2008. The power of the Test for Treatment Effects in Three-Level Cluster Randomized Designs. Journal of Research on Educational Effectiveness 1:66-88.

Konstantopoulos, S. 2006. The Power of the Test in Three-Level Designs.

Kramer, K. 1994. A modelling analysis of the effects of climatic warmings on the probability of spring frost damage to tree species in the Netherlands and Germany. Plant Cell Environment 17:367-377.

Kutschera, U., and K. J. Niklas. 2015. Darwin-Wallace Demons: Survival of the fastest in populations of duckweeds and the evolutionary history of an enigmatic group of angiosperms. Plant Biology $17: 24-32$.

Lacor, M. A. M. 1969. On the influence of gibberellic acid and kinetin on the germination of turions of Spirodela polyrhiza (L.) Schleiden. Acta Botanica Neerlandica 18:550-557.

Lemon, G. D., U. Posluszny, and B. C. Husband. 2001. Potential and realized rates of vegetative reproduction in Spirodela polyrhiza, Lemna minor, and Wolffia borealis. Aquatic Botany 70:79-87.

Ley, S., K. Dölger, and K. J. Appenroth. 1997. Carbohydrate metabolism as a possible physiological modulator of dormancy in turions of Spirodela polyrhiza (L.) Schleiden. Plant Science 129:1-7.

Li, J., L. Du, W. Guan, F. H. Yu, and M. van Kleunen. 2016. Latitudinal and longitudinal clines of phenotypic plasticity in the invasive herb Solidago canadensis in China. Oecologia 182:755-764. Springer Berlin Heidelberg.

Lieth, H. 1974. Phenology and Seasonality Modeling. Springer Science+Business Media, Chapel Hill, North Carolina.

Maccallum, R. C., M. W. Browne, and H. M. Sugawara. 1996. Power analysis and determination of sample size for covariance structure modeling of fit involving a particular measure of model. Psychological Methods 13:130-149.

Macel, M., T. Dostálek, S. Esch, A. Bucharová, N. M. van Dam, K. Tielbörger, K. J. F. Verhoeven, and Z. Münzbergová. 2017. Evolutionary responses to climate change in a range expanding plant. Oecologia 184:543-554. Springer Berlin Heidelberg.

Martínez-Ruiz, C., and E. M. García-Roger. 2015. Being first increases the probability of long diapause in rotifer resting eggs. Hydrobiologia 745:111-121.

Maxwell, C. S., and P. M. Magwene. 2017. When sensing is gambling: An experimental system reveals how plasticity can generate tunable bet-hedging strategies. Evolution 71:859-871.

Mejbel, H. S., and A. M. Simons. 2018. Aberrant clones: Birth order generates life history diversity in Greater Duckweed, Spirodela polyrhiza. Ecology and Evolution 8:2021-2031.

Morrongiello, J. R., N. R. Bond, D. A. Crook, and B. B. M. Wong. 2012. Spatial variation in egg size and egg number reflects trade-offs and bet-hedging in a freshwater fish. Journal of Animal Ecology 81:806817. 
Noh, S., E. R. Everman, C. M. Berger, and T. J. Morgan. 2017. Seasonal variation in basal and plastic cold tolerance: Adaptation is influenced by both long- and short-term phenotypic plasticity. Ecology and Evolution 7:5248-5257.

Norberg, J., M. C. Urban, M. Vellend, C. A. Klausmeier, and N. Loeuille. 2012. Eco-evolutionary responses of biodiversity to climate change. Nature Climate Change 2:747-751. Nature Publishing Group.

Oláh, V., A. Hepp, N. Y. Gaibor Vaca, M. Tamás, and I. Mészáros. 2018. Retrospective analyses of archive phytotoxicity test data can help in assessing internal dynamics and stability of growth in laboratory duckweed cultures. Aquatic Toxicology 201:40-46. Elsevier.

Oláh, V., A. Hepp, G. Lakatos, and I. Mészáros. 2014. Cadmium-induced turion formation of Spirodela polyrhiza (L.) Schleiden. Acta Biologica Szegediensis 58:103-108.

Oláh, V., A. Hepp, and I. Mészáros. 2015. Comparative study on the sensitivity of turions and active fronds of giant duckweed (Spirodela polyrhiza (L.) Schleiden) to heavy metal treatments. Chemosphere 132:40-46.

Oláh, V., A. Hepp, and I. Mészáros. 2017. Temporal dynamics in photosynthetic activity of Spirodela polyrhiza turions during dormancy release and germination. Environmental and Experimental Botany 136:50-58. Elsevier B.V.

Orzack, S. H., and E. Sober. 1994. How (not) to test an optimality model. Trends in Ecology and Evolution 9:265-267.

Perry, T. O. 1968. Dormancy, Turion Formation, and Germination by Different Clones of Spirodela polyrrhiza. Plant physiology 43:1866-1869.

Philippi, T., and J. Seger. 1989. Hedging one's evolutionary bets, revisited. Trends in Ecology and Evolution 4:41-44.

Price, T. D., A. Qvarnström, and D. E. Irwin. 2003. The role of phenotypic plasticity in driving genetic evolution. Proceedings of the Royal Society B: Biological Sciences 270:1433-1440.

Proulx, S. R., and T. Day. 2002. What can Invasion Analyses Tell us about Evolution under Stochasticity in Finite Populations? Selection 2:2-15.

Proulx, S. R., and H. Teotónio. 2017. What Kind of Maternal Effects Can Be Selected For in Fluctuating Environments? The American Naturalist 189:E118-E137.

Richardson, A. D., T. F. Keenan, M. Migliavacca, Y. Ryu, O. Sonnentag, and M. Toomey. 2013. Climate change, phenology, and phenological control of vegetation feedbacks to the climate system. Agricultural and Forest Meteorology 169:156-173. Elsevier B.V.

Richardson, B. A., L. Chaney, N. L. Shaw, and S. M. Still. 2016. Will phenotypic plasticity affecting flowering phenology keep pace with climate change? Global Change Biology 23:2499-2508.

Rigby, J. R., and A. Porporato. 2008. Spring frost risk in a changing climate. Geophysical Research Letters 35:1-5. 
Rossi, V., A. Gandolfi, and P. Menozzi. 2017. Mother's Age and Hatching Phenology Strategy of Heterocypris incongruens (Crustacea: Ostracoda) in Unpredictable Environment. Journal of Experimental Zoology Part A: Ecological Genetics and Physiology 325:701-712.

Saatkamp, A., L. Affre, T. Dutoit, and P. Poschlod. 2018. Plant traits and population characteristics predict extinctions in a long-term survey of Mediterranean annual plants. Biodiversity and Conservation 27:1-14. Springer Netherlands.

Scheiner, S. M. 1993. Genetics and evolution of phenotypic plasticity. journal of Annual Review of Ecology and Systematics. 24:35-68.

Schiffers, K., E. C. Bourne, S. Lavergne, W. Thuiller, and J. M. J. Travis. 2013. Limited evolutionary rescue of locally adapted populations facing climate change. Philosophical Transactions of the Royal Society B: Biological Sciences 368:0-9.

Schlichting, C. D., and M. Pigliucci. 1998. Phenotypic Evolution: A Reaction Norm Perspective. Sinauer, Sunderland, Mass.

Seger, J., and H. J. Brockmann. 1987. What is bet-hedging?

Simons, A. M. 2009. Fluctuating natural selection accounts for the evolution of diversification bet hedging. Proceedings of the Royal Society B: Biological Sciences 276:1987-1992.

Simons, A. M. 2011. Modes of response to environmental change and the elusive empirical evidence for bet hedging. Proceedings of the Royal Society B: Biological Sciences 278:1601-1609.

Simons, A. M. 2014. Playing smart vs. playing safe: The joint expression of phenotypic plasticity and potential bet hedging across and within thermal environments. Journal of Evolutionary Biology 27:1047-1056.

Simons, A. M., and M. O. Johnston. 2006. Environmental and genetic sources of diversification in the timing of seed germination: Implications for the evolution of bet hedging. Evolution; international journal of organic evolution 60:2280-2292.

Simons, A. M., and M. O. Johnston. 2003. Suboptimal timing of reproduction in Lobelia inflata may be a conservative bet-hedging strategy. J Evolution Biol 16:233-243.

Singh, V., B. Pandey, and S. Suthar. 2018. Phytotoxicity of amoxicillin to the duckweed Spirodela polyrhiza: Growth, oxidative stress, biochemical traits and antibiotic degradation. Chemosphere 201:492-502. Elsevier Ltd.

Starrfelt, J., and H. Kokko. 2012. Bet-hedging-a triple trade-off between means, variances and correlations. Biological Reviews 87:742-755.

Stinson, K. A. 2004. Natural selection favors rapid reproductive phenology in Potentilla pulcherrima (Rosaceae) at opposite ends of a subalpine snowmelt gradient. American Journal of Botany 91:531-539.

Stocker, T. F., D. Qin, G.-K. Plantter, M. Tignor, S. K. Allen, J. Boschung, A. Nauels, Y. Xia, V. Bex, P. M. 
Midgley, and (eds.). 2013. Climate Change 2013: The Physical Science Basis. Contribution of Working Group I to the Fifth Assessment Report of the Intergovernmental Panel on Climate Change. United Kingdom and New York, NY, USA.

Tarazona, E., E. M. García-Roger, and M. J. Carmona. 2017. Experimental evolution of bet hedging in rotifer diapause traits as a response to environmental unpredictability. Oikos 126:1162-1172.

Turelli, M. 1984. Heritable genetic variation via mutation-selection balance: Lerch's zeta meets the abdominal bristle. Theoretical Population Biology 25:138-193.

Umina, P. A., A. R. Weeks, M. R. Kearney, S. W. McKechnie, and A. A. Hoffmann. 2005. Evolution: A rapid shift in a classic clinal pattern in Drosophila reflecting climate change. Science 308:691-693.

van Kleunen, M., and M. Fischer. 2005. Constraints on the evolution of adaptive phenotypic plasticity in plants. The New phytologist 166:49-60.

Van Vuren, D., and K. B. Armitage. 1991. Duration of snow cover and its influence on life-history variation in yellow-bellied marmots. Canadian Journal of Zoology 69:1755-1758.

Vasseur, L., and L. W. Aarssen. 1992. Phenotypic plasticity in Lemna minor (Lemnaceae). Plant Systematics and Evolution 180:205-219.

Via, S., and R. Lande. 1985. Genotype-Environment interaction and the evolution of phenotypic plasticity. Evolution 39:505-522.

Wadgymar, S. M., J. E. Ogilvie, D. W. Inouye, A. E. Weis, and J. T. Anderson. 2018. Phenological responses to multiple environmental drivers under climate change: insights from a long-term observational study and a manipulative field experiment. New Phytologist 218:517-529.

Wang, G., and M. E. Dillon. 2014. Recent geographic convergence in diurnal and annual temperature cycling flattens global thermal profiles. Nature Climate Change 4:988-992.

Wang, W., G. Haberer, H. Gundlach, C. Gläßer, T. Nussbaumer, M. C. Luo, A. Lomsadze, M. Borodovsky, R. A. Kerstetter, J. Shanklin, D. W. Byrant, T. C. Mockler, K. J. Appenroth, J. Grimwood, J. Jenkins, J. Chow, C. Choi, C. Adam, X. H. Cao, J. Fuchs, I. Schubert, D. Rokhsar, J. Schmutz, T. P. Michael, K. F. X. Mayer, and J. Messing. 2014. The Spirodela polyrhiza genome reveals insights into its neotenous reduction fast growth and aquatic lifestyle. Nature Communications 5:1-13.

Wiens, J. J. 2016. Climate-Related Local Extinctions Are Already Widespread among Plant and Animal Species. PLoS Biology 14:1-18.

Ziegler, P., K. S. Sree, and K. J. Appenroth. 2017. The uses of duckweed in relation to water remediation. Desalination and Water Treatment 63:327-342. 